\title{
The impossibility of unbiased judgment aggregation
}

Citation for published version (APA):

Dietrich, F. K., \& List, C. (2007). The impossibility of unbiased judgment aggregation. METEOR, Maastricht University School of Business and Economics. METEOR Research Memorandum No. 022 https://doi.org/10.26481/umamet.2007022

Document status and date:

Published: 01/01/2007

DOI:

10.26481/umamet.2007022

Document Version:

Publisher's PDF, also known as Version of record

\section{Please check the document version of this publication:}

- A submitted manuscript is the version of the article upon submission and before peer-review. There can be important differences between the submitted version and the official published version of record.

People interested in the research are advised to contact the author for the final version of the publication, or visit the DOI to the publisher's website.

- The final author version and the galley proof are versions of the publication after peer review.

- The final published version features the final layout of the paper including the volume, issue and page numbers.

Link to publication

\footnotetext{
General rights rights.

- You may freely distribute the URL identifying the publication in the public portal. please follow below link for the End User Agreement:

www.umlib.nl/taverne-license

Take down policy

If you believe that this document breaches copyright please contact us at:

repository@maastrichtuniversity.nl

providing details and we will investigate your claim.
}

Copyright and moral rights for the publications made accessible in the public portal are retained by the authors and/or other copyright owners and it is a condition of accessing publications that users recognise and abide by the legal requirements associated with these

- Users may download and print one copy of any publication from the public portal for the purpose of private study or research.

- You may not further distribute the material or use it for any profit-making activity or commercial gain

If the publication is distributed under the terms of Article $25 \mathrm{fa}$ of the Dutch Copyright Act, indicated by the "Taverne" license above, 
Franz Dietrich, Christian List

The impossibility of unbiased judgment aggregation

$\mathrm{RM} / 07 / 022$

(RM/05/049 -revised-)

\section{METE@R}

Maastricht research school of Economics of TEchnology and ORganizations

Universiteit Maastricht

Faculty of Economics and Business Administration P.O. Box 616

NL - 6200 MD Maastricht

phone : ++31 433883830

fax $\quad$ : ++31433884873 



\title{
The impossibility of unbiased judgment aggregation
}

\author{
Franz Dietrich and Christian List ${ }^{1}$ \\ 9/2005, revised $6 / 2007$
}

Standard impossibility theorems on judgment aggregation over logically connected propositions either use a controversial systematicity condition or apply only to agendas of propositions with rich logical connections. Are there any serious impossibilities without these restrictions? We prove an impossibility theorem without systematicity that applies to most standard agendas: Every judgment aggregation function (with rational inputs and outputs) satisfying a condition called unbiasedness is dictatorial (or effectively dictatorial if we remove one of the agenda conditions). Our agenda conditions are tight. Applied illustratively to (strict) preference aggregation represented in our model, the result implies that every unbiased social welfare function with universal domain is effectively dictatorial.

Keywords: judgment aggregation, logic, impossibility, May's neutrality

\section{Introduction}

We prove a new impossibility theorem on the aggregation of individual judgments (acceptance or rejection) on logically connected propositions into corresponding collective judgments. Due to the flexible notion of a proposition, judgment aggregation can represent many realistic decision problems. For example, the propositions could be the following:

$a$ : "We can afford a budget deficit."

$a \rightarrow b$ : "If we can afford a budget deficit, then we should increase spending on education."

$b$ : "We should increase spending on education."

The interest in judgment aggregation was sparked by the observation that majority voting on logically connected propositions does not guarantee rational (i.e., complete and consistent) collective judgments: the "discursive paradox" (Pettit 2001). In our example, if individual judgments are as shown in Table 1, a majority accepts $a$, a majority accepts $a \rightarrow b$, and yet a majority rejects $b$.

Although judgment aggregation has many similarities to preference aggregation in Condorcet's and Arrow's tradition, judgment aggregation generalizes preference aggregation ${ }^{2}$ and faces additional complexities. A basic fact about Arrowian preference aggregation is the following. If, on a given agenda (here: set of alternatives under consideration), majority voting generates irrational

\footnotetext{
${ }^{1}$ F. Dietrich, Dept. of Quant. Economics, University of Maastricht, P.O. Box 616, 6200 MD Maastricht, The Netherlands; C. List, Dept. of Government, London School of Economics, London WC2A 2AE, U.K. We thank Klaus Nehring and the anonymous referees for comments on this paper.

${ }^{2}$ As illustrated below, preference aggregation can be formally represented as a special case of judgment aggregation by expressing preference relations as binary ranking propositions in predicate logic of the form $x P y$ (List and Pettit 2004, Dietrich and List 2007).
} 


\begin{tabular}{|c|c|c|c|}
\hline & $a$ & $a \rightarrow b$ & $b$ \\
\hline Individual 1 & True & True & True \\
\hline Individual 2 & True & False & False \\
\hline Individual 3 & False & True & False \\
\hline Majority & True & True & False \\
\hline
\end{tabular}

Table 1: A discursive paradox

collective preferences for some profiles of rational individual preferences (that is, if there are at least three alternatives), then so does any preference aggregation function satisfying Arrow's conditions (universal domain, weak Pareto, independence of irrelevant alternatives, non-dictatorship). Thus any agenda susceptible to Condorcet's paradox is, more generally, susceptible to Arrow's theorem. No such fact holds for judgment aggregation. Even if, on a given agenda (in this case: set of propositions under consideration), majority voting generates irrational collective judgments for some profiles of rational individual judgments (that is, if some set of at least three propositions is minimal inconsistent), other judgment aggregation functions may still guarantee collective rationality while satisfying the counterparts of Arrow's conditions. Thus an agenda susceptible to a discursive paradox need not be susceptible to the counterpart of Arrow's theorem. The above agenda is, like most example agendas in the literature, susceptible to a discursive paradox but not to an Arrow-stype impossibility. Neither the size of an agenda nor that of its minimal inconsistent subsets determines whether or not an Arrow-style impossibility applies. The logical interconnections between the propositions in the agenda matter in a surprisingly complex way. The recent literature on judgment aggregation has explored this complexity, which also constitutes the motivation for this paper.

List and Pettit $(2002,2004)$ formalized judgment aggregation and proved a first impossibility theorem, strengthened by Pauly and van Hees (2006), Dietrich (2007) and Dietrich and List (2007), which holds for most standard agendas, but imposes a strong condition of systematicity on the aggregation function. Systematicity is the conjunction of an Arrow-inspired independence condition (requiring propositionwise aggregation) and a global neutrality condition (requiring equal treatment of all propositions). Thus the price for the theorem's applicability to many agendas is the strength of its systematicity condition.

Given this problem, several authors have proved impossibility theorems in which systematicity is weakened to independence (Pauly and van Hees 2006, Dietrich 2006, Gärdenfors 2006, Nehring and Puppe 2006, van Hees 2007, Dietrich and List 2007, Dokow and Holzman 2005, Mongin 2005). But these results exclude many agendas, notably the one in the example above and many other standard agendas. This is not because the theorems have not been proved for these agendas, but because they do not hold for them. Indeed, Dokow and Holzman (2005), extending an earlier result by Nehring and Puppe (2002) in the related "property space" model, have identified the weakest agenda condi- 
tions for an (Arrow-style) impossibility with independence; and these agenda conditions are still rather strong.

Once we give up systematicity, are all serious impossibilities restricted to special agendas? Unfortunately not. We introduce a condition of unbiased aggregation, inspired by May's (1952) neutrality condition and much weaker than systematicity. Unbiasedness requires an equal treatment of each proposition and its negation, but not of any two propositions. For most agendas, every unbiased judgment aggregation function (with rational inputs and outputs) is dictatorial or at least effectively dictatorial. A mathematically related earlier result is a theorem by Nehring and Puppe (2005) on strategy-proof social choice functions that are neutral-within-issues, on which we comment later.

Our result is of interest in light of May's classic characterization of majority voting (1952), in so far as it shows that, as soon as May's binary agenda $\{p, \neg p\}$ is just slightly enriched by additional propositions, May's possibility turns into an impossibility even if May's monotonicity condition is dropped and anonymity is significantly weakened. Our result also applies to (strict) preference aggregation problems with three or more alternatives: every unbiased social welfare function with universal domain is effectively dictatorial. Further, our results can be applied to two related aggregation problems: the belief merging problem discussed in computer science (e.g., Konieczny and Pino-Perez 2002), and the aggregation of binary evaluations (e.g., Wilson 1975 and Dokow and Holzman 2005). Throughout this paper we adopt Dietrich's (2007) general logics framework (extending the model in List and Pettit 2002, 2004).

\section{Definitions}

Let $N=\{1,2, \ldots, n\}$ be a group of individuals $(n \geq 2)$ required to make collective judgments on logically connected propositions.

Language. We consider a language, given by a non-empty set $\mathbf{L}$ of sentences (propositions) closed under negation: $p \in \mathbf{L}$ implies $\neg p \in \mathbf{L}$, where $\neg$ denotes "not". In addition to negation, the language may contain any other logical operators needed to express the decision problem, such as the classical operators $\wedge$ ("and"), $\vee$ ("or") and $\rightarrow$ ("if-then"), modal operators, subjunctive conditionals, etc. As usual in logic, every set $S \subseteq \mathbf{L}$ is either consistent or inconsistent (not both). Our results require some regularity axioms on the consistency notion (valid for many logics, classical or non-classical, propositional or predicate): ${ }^{3}$

C1 Pairs $\{p, \neg p\} \subseteq \mathbf{L}$ are inconsistent (self-entailment)

C2 Subsets of consistent sets $S \subseteq \mathbf{L}$ are consistent (monotonicity).

\footnotetext{
${ }^{3}$ The conditions $\mathrm{C} 1-\mathrm{C} 3$ can be re-expressed in terms of (equivalent) conditions on the entailment relation in $\mathbf{L}$ (the conditions L1-L3 in Dietrich 2007, who uses the labels "I1-I3" for "C1-C3"). The reason is that a set $S \subseteq \mathbf{L}$ is consistent if and only if, for no $p \in \mathbf{L}, S$ entails $p$ and entails $\neg p$.
} 
C3 $\emptyset$ is consistent, and each consistent set $S \subseteq \mathbf{L}$ has a consistent superset $T$ $\subseteq \mathbf{L}$ containing a member of each pair $p, \neg p \in \mathbf{L}$ (completability).

For example, in a propositional logic, $\mathbf{L}$ contains propositions such as $a, b$, $a \rightarrow b, a \wedge b$, where $\{a, a \rightarrow b, \neg b\}$ is inconsistent, $\{a, \neg(a \wedge b)\}$ is consistent, etc. Various realistic decision problems can be represented in our model, including preference aggregation problems as illustrated below. Call a set $S \subseteq \mathbf{L}$ minimal inconsistent if it is inconsistent and every proper subset $T \subsetneq S$ is consistent. Call a proposition $p \in \mathbf{L}$ contingent if $\{p\}$ and $\{\neg p\}$ are consistent, a contradiction if $\{p\}$ is inconsistent, and a tautology if $\{\neg p\}$ is inconsistent.

Agenda. The agenda is a non-empty set $X \subseteq \mathbf{L}$ of propositions on which judgments are to be made, where $X$ is a union of pairs $\{p, \neg p\}$ (with $p$ not itself a negated proposition). If $X$ is infinite, we require the logic to be compact: every inconsistent set has a finite inconsistent subset (this holds for many logics). In the example above, the agenda is $X=\{a, \neg a, b, \neg b, a \rightarrow b, \neg(a \rightarrow b)\}$ in a propositional logic. Notationally, double negations cancel each other out (i.e., $\neg \neg p$ stands for $p){ }^{4}$ A subagenda of the agenda $X$ is a subset $Y \subseteq X$ that is itself an agenda, i.e., non-empty and a union of pairs $\{p, \neg p\}$.

Judgment sets. Each individual $i$ 's judgment set is a subset $A_{i} \subseteq X$, where $p \in A_{i}$ means that individual $i$ accepts proposition $p$. A judgment set $A_{i}$ is rational if it is (i) consistent and (ii) complete in the sense that, for every proposition $p \in X, p \in A_{i}$ or $\neg p \in A_{i}$. Let $\mathbf{U}$ be the set of all rational judgment sets. A profile is an $n$-tuple $\left(A_{1}, \ldots, A_{n}\right)$ of individual judgment sets.

Aggregation functions. A (judgment) aggregation function is a function $F$ that assigns to each profile $\left(A_{1}, \ldots, A_{n}\right)$ in some domain a collective judgment set $F\left(A_{1}, \ldots, A_{n}\right)=A \subseteq X$, where $p \in A$ means that the group accepts proposition $p$. An example is majority voting: here $F\left(A_{1}, \ldots, A_{n}\right)=\{p \in X$ : $\left.\left|\left\{i \in N: p \in A_{i}\right\}\right|>\left|\left\{i \in N: p \notin A_{i}\right\}\right|\right\}$ for all profiles $\left(A_{1}, \ldots, A_{n}\right)$ in the domain. All our results assume that the aggregation function is a function $F: \mathbf{U}^{n} \rightarrow \mathbf{U}$, that is:

- $F$ accepts as inputs all possible profiles $\left(A_{1}, \ldots, A_{n}\right)$ of rational individual judgment sets ("universal domain"), and

- $F$ generates as outputs rational collective judgment sets ("collective rationality").

This is a demanding rationality requirement on individuals and on the collective; but it is a standard requirement. Call aggregation function $F$ unanimitypreserving if $F(A, \ldots, A)=A$ for every unanimous profile $(A, \ldots, A)$ in the domain of $F$. Call an individual $i$ a dictator (and $F$ dictatorial) if $F\left(A_{1}, \ldots, A_{n}\right)=$ $A_{i}$ for all profiles $\left(A_{1}, \ldots, A_{n}\right)$ in the domain of $F$. For any subset $Y \subseteq X$, call in-

\footnotetext{
${ }^{4}$ Hereafter, when we write $\neg p$ and $p$ is already of the form $\neg q$, we mean $q$ (rather than $\neg \neg q)$.
} 
dividual $i$ a dictator on $Y$ (and $F$ dictatorial on $Y$ ) if $F\left(A_{1}, \ldots, A_{n}\right) \cap Y=A_{i} \cap Y$ for all profiles $\left(A_{1}, \ldots, A_{n}\right)$ in the domain of $F$.

\section{Unbiasedness}

Our central condition on an aggregation function is inspired by May's (1952) condition of neutrality:

Unbiasedness. For any proposition $p \in X$ and profiles $\left(A_{1}, \ldots, A_{n}\right),\left(A_{1}^{*}, \ldots\right.$, $\left.A_{n}^{*}\right)$ in the domain of $F$, if [for all individuals $i, p \in A_{i}$ if and only if $\neg p \in A_{i}^{*}$ ] then $\left[p \in F\left(A_{1}, \ldots, A_{n}\right)\right.$ if and only if $\left.\neg p \in F\left(A_{1}^{*}, \ldots, A_{n}^{*}\right)\right]$.

Unbiasedness requires an equal treatment of each proposition $p \in X$ and its negation $\neg p$, regardless of other judgments. If we decompose an aggregation problem into multiple decisions between proposition-negation pairs, it can be interpreted as the application of May's neutrality condition (1952) to each such pair. Unbiasedness is also related to Nehring and Puppe's (2005) neutrality-within-issues. ${ }^{5}$ Unbiasedness is considerably weaker than List and Pettit's (2002) condition of systematicity, which requires an aggregation function to be neutral between any two propositions $p, q \in X$ :

Systematicity. For any propositions $p, q \in X$ and profiles $\left(A_{1}, \ldots, A_{n}\right)$, $\left(A_{1}^{*}, \ldots, A_{n}^{*}\right)$ in the domain of $F$, if [for all individuals $i, p \in A_{i}$ if and only if $\left.q \in A_{i}^{*}\right]$ then $\left[p \in F\left(A_{1}, \ldots, A_{n}\right)\right.$ if and only if $\left.q \in F\left(A_{1}^{*}, \ldots, A_{n}^{*}\right)\right]$.

While systematicity permits all uniform propositionwise decision methods, such as majority voting, symmetrical supermajority voting, dictatorships or inverse dictatorships, unbiasedness also permits aggregation functions that apply different decision criteria to different propositions but the same criterion to each proposition $p \in X$ and its negation $\neg p$. For example, on some pairs $p, \neg p \in X$ one can apply majority voting, on others weighted majority voting, on yet others majority voting within some subgroup or dictatorships or erratic decision methods like inverse dictatorships, minority voting, or accepting a proposition if and only if it is supported by an odd number of individuals. Unbiasedness also differs from a global neutrality condition based on a permutation $\pi: X \rightarrow X$ of the agenda (e.g., van Hees 2007). It is by itself logically independent from independence, but implies independence under universal domain and collective rationality (see Lemma 1 below). Systematicity, by contrast, implies both independence and global neutrality.

\footnotetext{
${ }^{5} \mathrm{~A}$ neutral-within-issues and strategy-proof social choice function induces an unbiased and monotonic judgment aggregation function.
} 


\section{A first impossibility of unbiased aggregation}

Our theorems use two weak agenda conditions. Their precise form is justified by the tighness (necessity) of the conditions in our theorems, as shown below.

First, call agenda $X$ non-separable if it cannot be partitioned into two logically independent subagendas $X_{1}$ and $X_{2}$, each containing at least one contingent proposition (where $X_{1}$ and $X_{2}$ are logically independent if $B_{1} \cup B_{2}$ is consistent for any consistent subsets $B_{1} \subseteq X_{1}$ and $B_{2} \subseteq X_{2}$ ). Informally, non-separability requires that the decision problem cannot be split into two logically independent decision problems - a plausible condition in practice. The agenda in our example above and many others are non-separable. But if we extend our example agenda by adding a new pair $c, \neg c$, where $c$ is an atomic proposition, the new agenda is separable, namely into the old agenda and the binary agenda $\{c, \neg c\}$.

Second, call agenda $X$ minimally connected if it has these two properties: ${ }^{6}$

(i) It has a minimal inconsistent subset $Y$ of size at least three.

(ii) It has a minimal inconsistent subset $Y$ such that $(Y \backslash Z) \cup\{\neg p: p \in Z\}$ is consistent for some set $Z \subseteq Y$ of even size (the even-number negation condition in Dietrich 2007 and Dietrich and List 2007; equivalent, for finite $X$, to Dokow and Holzman's 2005 algebraic non-affineness condition).

All standard example agendas in the judgment aggregation literature are minimally connected, including our example agenda above (take $Y=\{a, a \rightarrow$ $b, \neg b\}$ in (i) and (ii) and $Z=\{a, \neg b\}$ ) and agendas representing preference aggregation problems with three or more alternatives (as discussed below). The notorious exception is $X=\{a, \neg a, b, \neg b, a \leftrightarrow b, \neg(a \leftrightarrow b)\}$, where $\leftrightarrow$ is taken to be a material biconditional (i.e., $a \leftrightarrow b$ is logically equivalent to $(a \wedge b) \vee$ $(\neg a \wedge \neg b)$ ): (ii) fails since $Z$ does not exist. However, even this agenda becomes minimally connected once $\leftrightarrow$ is taken to be a subjunctive biconditional, which is arguably more realistic (Dietrich forthcoming): take $Y=\{a, \neg b, a \leftrightarrow b\}$ in (i) and (ii) and $Z=\{a, a \leftrightarrow b\}$. Also the agenda $X$ assumed by List and Pettit (2002) - containing distinct atomic propositions $a, b$ and their conjunction $a \wedge b$ - is minimally connected: take $Y=\{a, b, \neg(a \wedge b)\}$ in (i) and (ii), and $Z=\{a, b\}$.

Theorem 1 For (and only for) a non-separable minimally connected agenda, every unbiased unanimity-preserving aggregation function $F: \mathbf{U}^{n} \rightarrow \mathbf{U}$ is dictatorial.

In Theorem 1, and also in Theorems 2 and 3 below, the necessity of the agenda conditions (the "only for") assumes a group of size $n \geq 3$. Theorem 1 implies, as we will see, the following corollary.

Corollary 1 Every unbiased unanimity-preserving aggregation function $F$ : $\mathbf{U}^{n} \rightarrow \mathbf{U}$ is dictatorial on each non-separable minimally connected subagenda.

\footnotetext{
${ }^{6}$ The sets $Y$ may or may not differ between parts (i) and (ii).
} 
By Theorem 1, for most agendas, all unbiased aggregation functions (with rational inputs and outputs) are degenerate: they are dictatorial or overrule unanimities. Thus non-degenerate aggregation functions must favour some propositions over their negations.

This theorem (and part of its proof) is related to Nehring and Puppe's (2005) results on strategy-proof social choice functions that are neutral-withinissues. Translated into our framework, their results imply that, under further weakened agenda conditions, every aggregation function $F: \mathbf{U}^{n} \rightarrow \mathbf{U}$ that is unbiased and monotonic (hence also unanimity-preserving) is dictatorial. So our result uses weaker aggregation conditions but stronger agenda conditions. Similar remarks apply also to our later theorems, which do not even require a unanimity condition.

To show the necessity part of Theorem 1 (the "only for"), which holds for group size $n \geq 3$, we simply specify counterexamples ("possibilities").

First, suppose agenda $X$ is separable, say into subagendas $X_{1}$ and $X_{2}$. Then an unbiased and unanimity-preserving but not dictatorial aggregation function $F: \mathbf{U}^{n} \rightarrow \mathbf{U}$ can be defined by

$$
F\left(A_{1}, \ldots, A_{n}\right)=F_{1}\left(A_{1} \cap X_{1}, \ldots, A_{n} \cap X_{1}\right) \cup F_{2}\left(A_{1} \cap X_{2}, \ldots, A_{n} \cap X_{2}\right),
$$

where $F_{1}$ and $F_{2}$ are dictatorships for the agendas $X_{1}$ and $X_{2}$, respectively, with a different dictator each time.

Next suppose $X$ violates part (i) of minimal connectedness; so all minimal inconsistent sets contain at most two propositions. Then majority voting among the first three individuals, given on the universal domain by

$$
F\left(A_{1}, \ldots, A_{n}\right)=\left\{p \in X: \text { a majority of } A_{1}, A_{2}, A_{3} \text { contains } p\right\}
$$

generates consistent (and complete) judgment sets, hence defines an unbiased unanimity-preserving aggregation function $F: \mathbf{U}^{n} \rightarrow \mathbf{U}$ that is not dictatorial.

Finally, suppose $X$ violates part (ii) of minimal connectedness. Then Dokow and Holzman's (2005) parity function among the first three individuals, defined on the universal domain by

$$
F\left(A_{1}, \ldots, A_{n}\right)=\left\{p \in X: \text { an odd number of } A_{1}, A_{2}, A_{3} \text { contains } p\right\}
$$

generates consistent (and complete) judgment sets (see Dokow and Holzman 2005; for a non-algebraic proof see Dietrich 2007). So $F$ defines an unbiased unanimity-preserving aggregation function $F: \mathbf{U}^{n} \rightarrow \mathbf{U}$ that is not dictatorial.

The sufficiency proof in Theorem 1 rests on some lemmas. We begin by establishing the standard property of "independence" or "propositionwise aggregation".

Independence. For any proposition $p \in X$ and profiles $\left(A_{1}, \ldots, A_{n}\right),\left(A_{1}^{*}, \ldots\right.$, $\left.A_{n}^{*}\right)$ in the domain of $F$, if [for all individuals $i, p \in A_{i}$ if and only if $p \in A_{i}^{*}$ ] then $\left[p \in F\left(A_{1}, \ldots, A_{n}\right)\right.$ if and only if $\left.p \in F\left(A_{1}^{*}, \ldots, A_{n}^{*}\right)\right]$. 
Lemma 1 Every unbiased aggregation function $F: \mathbf{U}^{n} \rightarrow \mathbf{U}$ is independent. ${ }^{7}$

Proof. Let $F$ be as specified. Consider any $p \in X$ and any profiles $\left(A_{1}, \ldots\right.$, $\left.A_{n}\right),\left(A_{1}^{*}, \ldots, A_{n}^{*}\right) \in \mathbf{U}^{n}$ in which the same set of individuals $C \subseteq N$ accepts $p$. We show that $p \in F\left(A_{1}, \ldots, A_{n}\right)$ if and only if $p \in F\left(A_{1}^{*}, \ldots, A_{n}^{*}\right)$, as required by independence. By collective rationality, if $p$ is a tautology, $p$ is contained in both $F\left(A_{1}, \ldots, A_{n}\right)$ and $F\left(A_{1}^{*}, \ldots, A_{n}^{*}\right)$; if $p$ is a contradiction, $p$ is contained in neither of $F\left(A_{1}, \ldots, A_{n}\right)$ and $F\left(A_{1}^{*}, \ldots, A_{n}^{*}\right)$. Suppose $p$ is contingent. Then $\neg p$ is also contingent. There exists a profile $\left(A_{1}^{\prime}, \ldots, A_{n}^{\prime}\right) \in \mathbf{U}^{n}$ such that exactly the individuals in $C$ accept $\neg p$. By unbiasedness, $p \in F\left(A_{1}, \ldots, A_{n}\right)$ is equivalent to $\neg p \in F\left(A_{1}^{\prime}, \ldots, A_{n}^{\prime}\right)$, which, again by unbiasedness, is equivalent to $p \in F\left(A_{1}^{*}, \ldots, A_{n}^{*}\right)$.

Using Lemma 1, we can easily see why Corollary 1 follows from Theorem 1. Let $F: \mathbf{U}^{n} \rightarrow \mathbf{U}$ be unbiased and unanimity-preserving. Consider a nonseparable minimally connected subagenda $X^{*} \subseteq X$. As $F$ is independent (by Lemma 1), $F$ induces a unique aggregation function $F^{*}$ for this subagenda: $F^{*}$ has universal domain (for agenda $\left.X^{*}\right)$, and is for all $\left(A_{1}^{*}, \ldots, A_{n}^{*}\right)$ in this domain given by

$$
F^{*}\left(A_{1}^{*}, \ldots, A_{n}^{*}\right)=F\left(A_{1}, \ldots, A_{n}\right) \cap X^{*},
$$

where $\left(A_{1}, \ldots, A_{n}\right)$ is a profile in $\mathbf{U}^{n}$ with $A_{i} \cap X^{*}=A_{i}^{*}$ for all $i$; by independence it does not matter which such profile $\left(A_{1}, \ldots, A_{n}\right)$ is chosen. The function $F^{*}$ inherits from $F$ the properties of unbiasedness, unanimity-preservation and collective rationality. So, by Theorem $1, F^{*}$ is dictatorial. Hence, by (1) and independence, $F$ is dictatorial on $X^{*}$, as desired.

For the next lemma, call propositions $p, q \in X$ connected (in $X$ ) if they are conditionally dependent: there exist $p^{*} \in\{p, \neg p\}$ and $q^{*} \in\{q, \neg q\}$ such that $\left\{p^{*}, q^{*}\right\} \cup Y$ is inconsistent for some $Y \subseteq X$ consistent with $p^{*}$ and with $q^{*}$. And call coalition $C \subseteq N$ winning for $p \in X$ (under $F$ ) if $p \in F\left(A_{1}, \ldots, A_{n}\right.$ ) for every profile $\left(A_{1}, \ldots, A_{n}\right)$ in the domain with $\left\{i: p \in A_{i}\right\}=C$. If an aggregation function $F$ is independent, it is uniquely determined by the family $\left(\mathcal{C}_{p}\right)_{p \in X}$, where $\mathcal{C}_{p}$ is the set of coalitions winning for $p \in X$. Specifically, for all profiles $\left(A_{1}, \ldots, A_{n}\right)$ in the domain,

$$
F\left(A_{1}, \ldots, A_{n}\right)=\left\{p \in X:\left\{i: p \in A_{i}\right\} \in \mathcal{C}_{p}\right\} .
$$

Lemma 2 Suppose $F: \mathbf{U}^{n} \rightarrow \mathbf{U}$ is unbiased. For any $p \in X$, let $\mathcal{C}_{p}$ be the set of coalitions winning for $p$. Then:

(a) If $p \in X$ is contingent, $\mathcal{C}_{p}=\mathcal{C}_{\neg p}$ and $C \in \mathcal{C}_{p} \Leftrightarrow N \backslash C \notin \mathcal{C}_{p}$.

(b) If $p, q \in X$ are connected and $F$ is unanimity-preserving, $\mathcal{C}_{p}=\mathcal{C}_{q}$.

\footnotetext{
${ }^{7}$ Unbiasedness does not imply independence without assuming universal domain and collective rationality.
} 
Proof. Let $F: \mathbf{U}^{n} \rightarrow \mathbf{U}$ be unbiased. By Lemma 1, $F$ is independent.

(a) Let $p \in X$ be contingent. To show $\mathcal{C}_{p}=\mathcal{C}_{\neg p}$, consider any $C \subseteq N$, and let us prove that $C \in \mathcal{C}_{p}$ if and only if $C \in \mathcal{C}_{\neg p}$. As $p$ is contingent, there exist profiles $\left(A_{1}, \ldots, A_{n}\right),\left(A_{1}^{*}, \ldots, A_{n}^{*}\right) \in \mathbf{U}^{n}$ such that $C=\left\{i: p \in A_{i}\right\}=\{i$ : $\left.\neg p \in A_{i}^{*}\right\}$. By unbiasedness, $p \in F\left(A_{1}, \ldots, A_{n}\right)$ if and only if $\neg p \in F\left(A_{1}^{*}, \ldots, A_{n}^{*}\right)$, whence $\left\{i: p \in A_{i}\right\} \in \mathcal{C}_{p}$ if and only if $\left\{i: \neg p \in A_{i}^{*}\right\} \in \mathcal{C}_{\neg p}$, i.e., $C \in \mathcal{C}_{p}$ if and only if $C \in \mathcal{C}_{\neg p}$.

To prove the second part of (a), let $C \subseteq N$ again. As $p$ is contingent, there exists a profile $\left(A_{1}, \ldots, A_{n}\right) \in \mathbf{U}^{n}$ such that $C=\left\{i: p \in A_{i}\right\}$, hence $N \backslash C=\left\{i: \neg p \in A_{i}\right\}$. Now $C \in \mathcal{C}_{p}$ is equivalent to $p \in F\left(A_{1}, \ldots, A_{n}\right)$, hence to $\neg p \notin F\left(A_{1}, \ldots, A_{n}\right)$, hence to $N \backslash C \notin \mathcal{C}_{\neg p}$, hence to $N \backslash C \notin \mathcal{C}_{p}$, as shown above.

(b) Suppose $p, q \in X$ are connected and $F$ is unanimity-preserving. Then there exist $v \in\{p, \neg p\}$ and $w \in\{q, \neg q\}$ and $Y \subseteq X$ such that (i) each of $\{v\} \cup Y$ and $\{w\} \cup Y$ is consistent, and (ii) $\{v, w\} \cup Y$ is inconsistent. It follows that (iii) each of $\{v, \neg w\} \cup Y$ and $\{\neg v, w\} \cup Y$ is consistent. By (iii), $v$ and $w$ are contingent. So, by part (a), it is sufficient to show that $\mathcal{C}_{v}=\mathcal{C}_{w}$. We only show that $\mathcal{C}_{v} \subseteq \mathcal{C}_{w}$, as the converse inclusion is analogous. Suppose $C \in \mathcal{C}_{v}$. By (iii) there exists a profile $\left(A_{1}, \ldots, A_{n}\right) \in \mathbf{U}^{n}$ such that $\{v, \neg w\} \cup Y \subseteq A_{i}$ for all $i \in C$ and $\{\neg v, w\} \cup Y \subseteq A_{i}$ for all $i \in N \backslash C$. We have $v \in F\left(A_{1}, \ldots, A_{n}\right)$ by $C \in \mathcal{C}_{v}$, and $Y \subseteq F\left(A_{1}, \ldots, A_{n}\right)$ by $N \in \mathcal{C}_{v}$. By $\{v\} \cup Y \subseteq F\left(A_{1}, \ldots, A_{n}\right)$ and (ii), $w \notin F\left(A_{1}, \ldots, A_{n}\right)$. So $N \backslash C \notin \mathcal{C}_{w}$, and hence $C \in \mathcal{C}_{w}$ by part (a), as required.

We now show that non-separability of the agenda $X$ is equivalent to another structural property. Call propositions $p, q \in X$ indirectly connected if there exist $p_{1}, \ldots, p_{k} \in X$ with $p_{1}=p$ and $p_{k}=q$ such that any two neighbours $p_{t}, p_{t+1}$ are connected (as defined above). And call agenda $X$ indirectly connected if any two contingent propositions $p, q \in X$ are indirectly connected.

Lemma 3 An agenda is non-separable if and only if it is indirectly connected.

Proof. We may assume without loss of generality that all $p \in X$ are contingent, because if $X$ also has non-contingent members then $X$ is indirectly connected if and only if the subagenda $\{p \in X: p$ is contingent $\}$ is, and $X$ is non-separable if and only if $\{p \in X: p$ is contingent $\}$ is (if $X$ has only non-contingent members, the claim is trivial).

First, assume $X$ is separable. Then there is a partition of $X$ into logically independent subagendas $X_{1}, X_{2}$. Consider any $p \in X_{1}$ and $q \in X_{2}$. We show that $p$ and $q$ are not indirectly connected. Suppose for a contradiction that $p_{1}, \ldots, p_{m} \in X(m \geq 1)$ are such that $p=p_{1}, q=p_{m}$, and $p_{t}$ and $p_{t+1}$ are connected for any $t \in\{1, \ldots, m-1\}$. As $p_{1} \in X_{1}$ and $p_{m} \in X_{2}$, there must be a $t \in\{1, \ldots, m-1\}$ such that $p_{t} \in X_{1}$ and $p_{t+1} \in X_{2}$. As $p_{t}$ and $p_{t+1}$ are connected, there are $p_{t}^{*} \in\left\{p_{t}, \neg p_{t}\right\}, p_{t+1}^{*} \in\left\{p_{t+1}, \neg p_{t+1}\right\}$ and $Y \subseteq X$ such that (i) $\left\{p_{t}^{*}, p_{t+1}^{*}\right\} \cup Y$ is inconsistent and (ii) each of $\left\{p_{t}^{*}\right\} \cup Y$ and $\left\{p_{t+1}^{*}\right\} \cup Y$ is consistent. By (ii), each of the sets $B_{1}:=\left(\left\{p_{t}^{*}\right\} \cup Y\right) \cap X_{1}$ and $B_{1}:=$ 
$\left(\left\{p_{t+1}^{*}\right\} \cup Y\right) \cap X_{2}$ is consistent. So $B_{1} \cup B_{2}$ is consistent, as $X_{1}$ and $X_{2}$ are logically independent. But

$$
\begin{aligned}
B_{1} \cup B_{2} & =\left[\left(\left\{p_{t}^{*}\right\} \cup Y\right) \cap X_{1}\right] \cup\left[\left(\left\{p_{t+1}^{*}\right\} \cup Y\right) \cap X_{2}\right] \\
& =\left[\left(\left\{p_{t}^{*}, p_{t+1}^{*}\right\} \cup Y\right) \cap X_{1}\right] \cup\left[\left(\left\{p_{t}^{*}, p_{t+1}^{*}\right\} \cup Y\right) \cap X_{2}\right] \\
& =\left(\left\{p_{t}^{*}, p_{t+1}^{*}\right\} \cup Y\right) \cap\left[X_{1} \cup X_{2}\right]=\left\{p_{t}^{*}, p_{t+1}^{*}\right\} \cup Y,
\end{aligned}
$$

which is inconsistent by (ii), a contradiction.

Secondly, let $X$ be not indirectly connected. We show that $X$ is separable. By assumption, there exist $p, q \in X$ that are not indirectly connected. Define $X_{1}:=\{r \in X: p$ and $r$ are indirectly connected $\}$ and $X_{2}:=X \backslash X_{1}$. Since $p$ is indirectly connected to itself (as $p$ is contingent), $p \in X_{1}$. Further, $q \in X_{2}$. So each of $X_{1}$ and $X_{2}$ is non-empty. Moreover, each of $X_{1}$ and $X_{2}$ is closed under negation. If follows that $X_{1}$ and $X_{2}$ are subagendas of $X$.

We complete the proof by showing that $X_{1}$ and $X_{2}$ are logically independent. Suppose for a contradiction that $B_{1} \subseteq X_{1}$ and $B_{2} \subseteq X_{2}$ are each consistent but that $B_{1} \cup B_{2}$ is inconsistent. As $X$ is finite or the logic compact, there exists a minimal inconsistent subset $B \subseteq B_{1} \cup B_{2}$. We have neither $B \subseteq B_{1}$ nor $B \subseteq B_{2}$, since otherwise $B$ would be consistent. So there exist $r \in B \cap X_{1}$ and $s \in B \cap X_{2}$. $r$ and $s$ are connected, because, putting $Y:=B \backslash\{r, s\},\{r, s\} \cup Y=B$ is inconsistent, but each of $\{r\} \cup Y=B \backslash\{s\}$ and $\{s\} \cup Y=B \backslash\{r\}$ is consistent by $B$ 's minimal inconsistency. This is a contradiction.

Essentially by combining Lemma 3 with part (b) of Lemma 2, we now deduce systematicity (which brings us into the terrain of known results of the literature).

Lemma 4 For a non-separable agenda $X$, every unbiased unanimity-preserving aggregation function $F: \mathbf{U}^{n} \rightarrow \mathbf{U}$ is systematic.

Proof. Let $X$ and $F$ be as specified. Consider propositions $p, q \in X$ and profiles $\left(A_{1}, \ldots, A_{n}\right),\left(A_{1}^{*}, \ldots, A_{n}^{*}\right) \in \mathbf{U}^{n}$, such that $(*)$ for all individuals $i$, $p \in A_{i} \Leftrightarrow q \in A_{i}^{*}$. We show that $\left({ }^{* *}\right) p \in F\left(A_{1}, \ldots, A_{n}\right) \Leftrightarrow q \in F\left(A_{1}^{*}, \ldots, A_{n}^{*}\right)$. If $p$ and $q$ are both contingent, then they are indirectly connected by Lemma 3 , hence have the same set of winning coalitions by iterated applications of part (b) of Lemma 2; so, using $\left({ }^{*}\right)$, we obtain $\left(^{* *}\right)$. If $p$ or $q$ is a tautology then, by individual rationality and $\left(^{*}\right)$, all $A_{i}$ contain $p$ and all $A_{i}^{*}$ contain $q$; so by unanimitypreservation (and independence) $p \in F\left(A_{1}, \ldots, A_{n}\right)$ and $q \in F\left(A_{1}^{*}, \ldots, A_{n}^{*}\right)$, implying $(* *)$. Finally, if $p$ or $q$ is a contradiction then, by individual rationality and $\left(^{*}\right)$, all $A_{i}$ contain $\neg p$ and all $A_{i}^{*}$ contain $\neg q$; so by unanimity-preservation (and independence) $\neg p \in F\left(A_{1}, \ldots, A_{n}\right)$ and $\neg q \in F\left(A_{1}^{*}, \ldots, A_{n}^{*}\right)$, which again implies (**) (by collective rationality).

Lemma 4 together with the following known result implies the sufficiency part of Theorem 1, hence completes the proof. 
Lemma 5 (Dietrich and List 2007) For a minimally connected agenda, every systematic unanimity-preserving aggregation function $F: \mathbf{U}^{n} \rightarrow \mathbf{U}$ is dictatorial.

To make the present argument self-contained, we sketch the proof of this result. Let the agenda $X$ and the function $F$ be as specified. By systematicity, there is a set $\mathcal{C}$ of ("winning") coalitions $C \subseteq N$ such that

$$
F\left(A_{1}, \ldots, A_{n}\right)=\left\{p \in X:\left\{i: p \in A_{i}\right\} \in \mathcal{C}\right\} \text { for all }\left(A_{1}, \ldots, A_{n}\right) \in \mathbf{U}^{n} .
$$

Steps (a)-(e) below show the existence of a dictator $i$, i.e., $\mathcal{C}=\{C \subseteq N: i \in C\}$.

(a) $N \in \mathcal{C}$, by unanimity-preservation.

(b) $C \in \mathcal{C} \Leftrightarrow N \backslash C \notin \mathcal{C}$, as exactly one member of any $p, \neg p \in X$ is accepted.

(c) $\left[C \in \mathcal{C} \& C \subseteq C^{*} \subseteq N\right] \Rightarrow C^{*} \in \mathcal{C}$. Suppose $C \in \mathcal{C} \& C \subseteq C^{*} \subseteq N$. Let $Y \subseteq X$ be as in part (ii) of "minimal connectedness". For illustrative purposes, let $Y$ be binary, say $Y=\{p, q\}$ (see Dietrich and List 2007 for the general case). Then $\{p, q\}$ is inconsistent, but $\{p, \neg q\},\{\neg p, q\},\{\neg p, \neg q\}$ are each consistent. Consider a profile $\left(A_{1}, \ldots, A_{n}\right) \in \mathbf{U}^{n}$ such that $\{p, \neg q\} \subseteq A_{i}$ if $i \in C,\{\neg p, \neg q\} \subseteq A_{i}$ if $i \in C^{*} \backslash C$, and $\{\neg p, q\} \subseteq A_{i}$ otherwise. As $C \in \mathcal{C}$, $p \in F\left(A_{1}, \ldots, A_{n}\right)$. So, by $\{p, q\}$ 's inconsistency, $\neg q \in F\left(A_{1}, \ldots, A_{n}\right)$. Hence $C^{*} \in \mathcal{C}$.

(d) $C, C^{*} \in \mathcal{C} \Rightarrow C \cap C^{*} \in \mathcal{C}$. Let $C, C^{*} \in \mathcal{C}$. Let $Y \subseteq X$ be as in part (i) of "minimal connectedness". Consider distinct $p, q, r \in Y$. Consider a profile in which each individual accepts all $s \in Y \backslash\{p, q, r\}$, and rejects exactly one of $p, q, r$ : all $i \in C \cap C^{*}$ reject $p$, all $i \in C^{*} \backslash C$ reject $q$, and all others reject $r$. By (a), all $s \in Y \backslash\{p, q, r\}$ are collectively accepted. As exactly all $i \in C^{*}(\in \mathcal{C})$ accept $r$, the collective accepts $r$. As at least all $i \in C(\in \mathcal{C})$ accept $q$, the collective accepts $q$ by (c). So, as $Y$ is inconsistent, the collective accepts $\neg p$. Hence $C \cap C^{*} \in \mathcal{C}$.

(e) $\mathcal{C}=\{C \subseteq N: i \in C\}$ for some individual (dictator) $i$. By (d), $\tilde{C}:=$ $\cap_{C \in \mathcal{C}} C \in \mathcal{C}$. So $\tilde{C} \neq \emptyset$ (as $\emptyset \notin \mathcal{C}$ by (a) and (b)). $\tilde{C}$ must be singleton, say $\tilde{C}=\{i\}$ : otherwise $\tilde{C}$ could be partitioned into two non-empty coalitions $C, C^{\prime}$, where $N \backslash C, N \backslash C^{\prime} \in \mathcal{C}$ by (b), hence $N \backslash \tilde{C}=(N \backslash C) \cap\left(N \backslash C^{\prime}\right) \in \mathcal{C}$ by (d), and so $\tilde{C} \notin \mathcal{C}$ by (b), a contradiction. By $\{i\} \in \mathcal{C}$ and (c), $\{C \subseteq N: i \in C\} \subseteq \mathcal{C}$. This inclusion also implies the converse inclusion by (b).

\section{Two impossibilities without the unanimity condition}

To further strengthen the impossibility of unbiased aggregation, we now show that even without requiring unanimity-preservation we still run into impossibility. We prove two theorems, both of which merely assume the aggregation function $F: \mathbf{U}^{n} \rightarrow \mathbf{U}$ to be unbiased. In the first result, we strengthen Theorem 1's agenda condition in such a way that unanimity-preservation can be 
derived; so Theorem 1 applies and implies dictatorship. In the second result, we keep Theorem 1's mild agenda condition, and show that an equally undesirable variant of dictatorship follows: an "effective dictatorship".

Given that we now only require the aggregation function $F: \mathbf{U}^{n} \rightarrow \mathbf{U}$ to be unbiased, it may seem that at least some such $F$ can overrule at least some unanimous judgments. Surprisingly, for many agendas this is not the case. First assume the agenda $X$ is asymmetric: there exists a consistent set $A \subseteq X$ such that $\{\neg p: p \in A\}$ is inconsistent (Dietrich 2007, Dietrich and List 2007). Then $F$ cannot overrule unanimous judgments for every $p \in A$ : otherwise, if all individuals accept all $p \in A$, the collective accepts all $\neg p$ (with $p \in A$ ), a collective inconsistency. But $F$ may still overrule unanimous judgments on some $p \in X \backslash A$. To prevent any overruling of unanimity, the agenda must be locally asymmetric: for every subagenda $Y \subseteq X$, there exists a consistent set $A \subseteq X$ that becomes inconsistent by negating those propositions that are in $Y$ (i.e., $(A \backslash Y) \cup\{\neg p: p \in A \cap Y\}$ is inconsistent). Examples are discussed shortly.

Lemma 6 For (and only) for a locally asymmetric agenda, every unbiased aggregation function $F: \mathbf{U}^{n} \rightarrow \mathbf{U}$ is unanimity-preserving.

Proof. First suppose $F: \mathbf{U}^{n} \rightarrow \mathbf{U}$ is unbiased and not unanimity-preserving. Then $Z:=\{p \in X: N$ is not a winning coalition for $p\}$ is non-empty. $Z$ is closed under negation: if $p \in Z$ then $p$ is contingent, and hence $\neg p \in Z$ by part (a) of Lemma 2. So $Z$ is a subagenda. To show that $X$ is not locally asymmetric, we show that, for every consistent set $A \subseteq X$, also $(A \backslash Z) \cup\{\neg p: p \in A \cap Z\}$ is consistent. It obviously suffices to show this for the case that $A$ is complete. So let $A \subseteq X$ be complete and consistent, i.e., $A \in \mathrm{U}$. By definition of $Z$, $F(A, \ldots, A)$ contains all $p \in A \backslash Z$ and all $\neg p$ with $p \in A \cap Z$, the latter because by part (a) of Lemma 2 the empty coalition is winning for $\neg p$. So $F(A, \ldots, A)$ contains all members of the set $(A \backslash Z) \cup\{\neg p: p \in A \cap Z\}$. Hence this set is consistent, as desired.

Conversely, suppose $X$ is not locally asymmetric. Then there is a subagenda $Z \subseteq X$ such that $\left(^{*}\right)$ for all consistent sets $A \subseteq X$, also $(A \backslash Z) \cup\{\neg p: p \in A \cap Z\}$ consistent. Let $i$ be an individual and $F$ the aggregation function with universal domain $\mathbf{U}^{n}$ that makes $i$ a dictator on $X \backslash Z$ and an inverse dictator on $Z$; that is, for all $\left(A_{1}, \ldots, A_{n}\right) \in \mathbf{U}^{n}$,

$$
F\left(A_{1}, \ldots, A_{n}\right)=\left(A_{i} \backslash Z\right) \cup\left\{\neg p: p \in A_{i} \cap Z\right\} .
$$

By construction, $F$ is unbiased and generates complete judgment sets. It also generates consistent ones by $\left(^{*}\right)$; hence $F: \mathbf{U}^{n} \rightarrow \mathbf{U}$. But, as desired, $F$ is not unanimity-preserving, because for all $(A, \ldots, A) \in \mathbf{U}^{n}$ we have $F(A, \ldots, A)=$ $(A \backslash Z) \cup\{\neg p: p \in A \cap Z\} \neq A$.

This lemma together with Theorem 1 implies our next theorem. 
Theorem 2 For (and only for) a non-separable, minimally connected and locally asymmetric agenda, every unbiased aggregation function $F: \mathbf{U}^{n} \rightarrow \mathbf{U}$ is dictatorial.

As for Theorem 1, a corollary follows (using Lemma 1):

Corollary 2 Every unbiased aggregation function $F: \mathbf{U}^{n} \rightarrow \mathbf{U}$ is dictatorial on each non-separable, minimally connected and locally asymmetric subagenda.

Theorem 2 applies to our example agenda $X=\{a, \neg a, b, \neg b, a \rightarrow b, \neg(a \rightarrow$ $b)\}$, which is locally asymmetric, as we now check by going through the subagendas $Y \subseteq X$. For $Y=\{a, \neg a\}$, let $A=\{\neg a, a \rightarrow b, \neg b\}$ (as $A$ is consistent but $\{a, a \rightarrow b, \neg b\}$ is not). For $Y=\{b, \neg b\}$, let $A=\{a, a \rightarrow b, b\}$. In general, for any $Y$, let $A$ be the set that becomes $\{a, a \rightarrow b, \neg b\}$ by negating all $p \in Y$, i.e., the set arising from $\{a, a \rightarrow b, \neg b\}$ by negating all $p \in Y$; this $A$ is indeed consistent since $\{a, a \rightarrow b, \neg b\}$ becomes consistent by negating any member(s), assuming here that " $\rightarrow$ " is a subjunctive implication (see Dietrich forthcoming). If, less realistically, " $\rightarrow$ " is a material implication (i.e., $a \rightarrow b$ is equivalent to $\neg a \vee b$ ), other choices of $A$ work.

But Theorem 2 does not apply to the strict preference aggregation problem discussed below: this agenda is not locally asymmetric, in fact not even asymmetric simpliciter because inverting (negating) rational strict preferences yields rational strict preferences. This illustrates that the strong impossibility of Theorem 2 ("unbiasedness implies dictatorship") applies to fewer agendas than the impossibilities of Theorem 1 above and Theorem 3 below.

Now we turn to our last impossibility result, which requires neither the unanimity condition of Theorem 1 nor the extra agenda condition of Theorem 2 .

For any subset $Y \subseteq X$, call individual $i$ an inverse dictator on $Y$ if $F\left(A_{1}, \ldots, A_{n}\right) \cap Y=Y \backslash A_{i}$ for all profiles $\left(A_{1}, \ldots, A_{n}\right)$ in the domain. Call individual $i$ an effective dictator (and $F$ effectively dictatorial) if there is a partition of $X$ into subsets $X_{+}, X_{-}$(each one possibly empty) such that individual $i$ is a dictator on $X_{+}$and an inverse dictator on $X_{-}$; that is, $F\left(A_{1}, \ldots, A_{n}\right)=$ $\left(X_{+} \cap A_{i}\right) \cup\left(X_{-} \backslash A_{i}\right)$ for all profiles $\left(A_{1}, \ldots, A_{n}\right)$ in the domain. For any subset $Y \subseteq X$, call individual $i$ an effective dictator on $Y$ (and $F$ effectively dictatorial on $Y$ ) if there is a partition of $Y$ into subsets $Y_{+}, Y_{-}$(each one possibly empty) such that individual $i$ is a dictator on $Y_{+}$and an inverse dictator on $Y_{-}$; that is, $F\left(A_{1}, \ldots, A_{n}\right) \cap Y=\left(Y_{+} \cap A_{i}\right) \cup\left(Y_{-} \backslash A_{i}\right)$ for all profiles $\left(A_{1}, \ldots, A_{n}\right)$ in the domain.

Theorem 3 For (and only for) a non-separable minimally connected agenda, every unbiased aggregation function $F: \mathbf{U}^{n} \rightarrow \mathbf{U}$ is effectively dictatorial.

Again, a corollary follows (using Lemma 1): 
Corollary 3 Every unbiased aggregation function $F: \mathbf{U}^{n} \rightarrow \mathbf{U}$ is effectively dictatorial on each non-separable minimally connected subagenda.

Note a further corollary. Call aggregation function $F$ anonymous if, for any profiles $\left(A_{1}, \ldots, A_{n}\right)$ and $\left(A_{\sigma(1)}, \ldots, A_{\sigma(n)}\right)$ in the domain, where $\sigma: N \rightarrow N$ is a permutation, $F\left(A_{1}, \ldots, A_{n}\right)=F\left(A_{\sigma(1)}, \ldots, A_{\sigma(n)}\right)$.

Corollary 4 If the agenda has a non-separable and minimally connected subagenda (possibly the agenda itself), there exists no anonymous unbiased aggregation function $F: \mathbf{U}^{n} \rightarrow \mathbf{U}$.

Corollary 4 significantly strengthens List and Pettit's (2002) theorem by weakening systematicity to unbiasedness and weakening the agenda condition. Without weakening systematicity to unbiasedness, the agenda condition in List and Pettit's result can be weakened further than in Corollary 4, namely to minimal connectedness alone (Dietrich and List 2007).

Theorem 3 and Corollary 4 are particularly interesting in light of May's classic characterization of majority voting (1952). Translated into our terminology, May's theorem (without indifference) states that, for any binary agenda $X=\{p, \neg p\}$, an aggregation function $F: \mathbf{U}^{n} \rightarrow \mathbf{U}$ is majority voting if and only if it is anonymous, unbiased and monotonic. ${ }^{8}$ Our result shows that, if the agenda is just slightly enriched beyond binariness, May's theorem collapses into an impossibility result even if monotonicity is dropped and anonymity is weakened to the requirement that there be no effective dictator.

In Theorem 3, the necessity of the agenda conditions (the "only for") follows from the aggregation functions constructed earlier to show necessity in Theorem 1. These aggregation functions are not only not dictatorial but also not effectively dictatorial.

Regarding sufficiency, we first show that the aggregation function $F$ in Theorem 3 induces a unanimity-preserving aggregation function $\widehat{F}$ :

Lemma 7 Suppose $F: \mathbf{U}^{n} \rightarrow \mathbf{U}$ is unbiased. Define, for each $p \in X$,

$$
\widehat{p}:= \begin{cases}p & \text { if } N \text { is a winning coalition for } p \\ \neg p & \text { if } N \text { is not a winning coalition for } p .\end{cases}
$$

Then:

(a) For any $p \in X, \widehat{\neg p}=\neg \widehat{p}$ and $\widehat{\widehat{p}}=p$.

(b) For any $A \subseteq X, A$ is consistent if and only if $\{\widehat{p}: p \in A\}$ is consistent.

(c) The aggregation function $\widehat{F}$ with universal domain $\mathbf{U}^{n}$ defined by

$$
\widehat{F}\left(A_{1}, \ldots, A_{n}\right)=\left\{\widehat{p}: p \in F\left(A_{1}, \ldots, A_{n}\right)\right\}
$$

satisfies unbiasedness, unanimity-preservation and collective rationality.

${ }^{8}$ Where $n$ is odd. An aggregation function $F$ is monotonic if, for all individuals $i$ and all profiles $\left(A_{1}, \ldots, A_{n}\right),\left(A_{1}, \ldots, A_{i}^{*}, \ldots, A_{n}\right)$ in the domain differing only in $i$ 's judgment set, if $F\left(A_{1}, \ldots, A_{n}\right)=A_{i}^{*}$ then $F\left(A_{1}, \ldots, A_{i}^{*}, \ldots, A_{n}\right)=A_{i}^{*}$. 
(d) For every $p \in X$,

$$
\mathcal{C}_{p}= \begin{cases}\widehat{\mathcal{C}_{p}} & \text { if } \widehat{p}=p \\ \left\{C \subseteq N: C \notin \widehat{\mathcal{C}_{p}}\right\} & \text { if } \widehat{p}=\neg p,\end{cases}
$$

where $\mathcal{C}_{p}\left(\widehat{\mathcal{C}_{p}}\right)$ is the set of coalitions winning for $p$ under $F(\widehat{F})$.

Proof. Let $F$ be as specified.

(a) Suppose $p \in X$. N is winning for $p$ if and only if $N$ is winning for $\neg p$; if $p$ is contingent this follows easily from unbiasedness (see also part (a) of Lemma 2 ); if $p$ is not contingent it holds because $N$ is winning for every tautology and (vacuously) for every contradiction. As $N$ is winning for $p$ if and only if $N$ is winning for $\neg p$, we have $\widehat{p}=p$ if and only if $\widehat{\neg p}=\neg p$, whence $\widehat{\neg p}=\neg \widehat{p}$. Moreover, if $\widehat{p}=p$ then $\widehat{\widehat{p}}=\widehat{p}=p$, and if $\widehat{p}=\neg p$ then $\widehat{\widehat{p}}=\widehat{\neg p}=\neg \widehat{p}=\neg \neg p=p$.

(b) Let $A \subseteq X$. By (a) it is sufficient to show one direction of the implication. Let $A$ be consistent. Then there exists a set $B \in \mathbf{U}$ such that $A \subseteq B$. For each $p \in A, F(B, \ldots, B)$ contains $\widehat{p}$ because:

- if $N \in \mathcal{C}_{p}$ then $\widehat{p}=p \in F(B, \ldots, B)$;

- if $N \notin \mathcal{C}_{p}$ then $p \notin F(B, \ldots, B)$, and so $\widehat{p}=\neg p \in F(B, \ldots, B)$.

By $\{\widehat{p}: p \in A\} \subseteq F(B, \ldots, B),\{\widehat{p}: p \in A\}$ is consistent.

(c) For any $\left(A_{1}, \ldots, A_{n}\right) \in \mathbf{U}^{n}, \widehat{F}\left(A_{1}, \ldots, A_{n}\right)$ is

- consistent by (b) and the consistency of $F\left(A_{1}, \ldots, A_{n}\right)$;

- complete as, for any $p \in X$, if $p \notin \widehat{F}\left(A_{1}, \ldots, A_{n}\right)$ then $\widehat{p} \notin F\left(A_{1}, \ldots, A_{n}\right)$ by $p=\widehat{\widehat{p}}$, hence $\neg \widehat{p} \in F\left(A_{1}, \ldots, A_{n}\right)$, and so $\widehat{F}\left(A_{1}, \ldots, A_{n}\right)$ contains $\widehat{\neg \widehat{p}}=\widehat{\widehat{\neg p}}=\neg p$.

$\widehat{F}$ is unanimity-preserving: for any $p \in X$ and any $\left(A_{1}, \ldots, A_{n}\right) \in \mathbf{U}^{n}$ with $p \in A_{i}$ for all individuals $i$,

- if $p \in F\left(A_{1}, \ldots, A_{n}\right)$, then $N$ is a winning coalition for $p$ under $F$ by independence (see Lemma 1 ), hence $\widehat{p}=p$, and so $p \in \widehat{F}\left(A_{1}, \ldots, A_{n}\right)$;

- if $p \notin F\left(A_{1}, \ldots, A_{n}\right)$, then $\neg p \in F\left(A_{1}, \ldots, A_{n}\right)$, hence $\widehat{\neg p} \in \widehat{F}\left(A_{1}, \ldots, A_{n}\right)$, where $\widehat{\neg p}=\neg \widehat{p}=\neg \neg p=p$ (since $\widehat{p}=\neg p$ ).

To show that $\widehat{F}$ is unbiased, consider any $p \in X$ and $\left(A_{1}, \ldots, A_{n}\right),\left(A_{1}^{*}, \ldots, A_{n}^{*}\right)$ $\in \mathbf{U}^{n}$ such that $p \in A_{i}$ if and only if $\neg p \in A_{i}^{*}$. Then $(*) \widehat{p} \in A_{i}$ if and only if $\neg \widehat{p} \in A_{i}^{*}$. Now $p \in \widehat{F}\left(A_{1}, \ldots, A_{n}\right)$ is equivalent to $\widehat{p} \in F\left(A_{1}, \ldots, A_{n}\right)$, by definition of $\widehat{F}$ and as $p=\widehat{\hat{p}}$. The latter is equivalent to $\neg \widehat{p} \in F\left(A_{1}^{*}, \ldots, A_{n}^{*}\right)$, by $\left(^{*}\right)$ and as $F$ is unbiased. This, in turn, is equivalent to $\widehat{\neg \hat{p}} \in \widehat{F}\left(A_{1}^{*}, \ldots, A_{n}^{*}\right)$ by definition of $\widehat{F}$, i.e., to $\neg p \in \widehat{F}\left(A_{1}^{*}, \ldots, A_{n}^{*}\right)$ as $\widehat{\neg \widehat{p}}=\widehat{\widehat{\neg p}}=\neg p$ by part (a).

(d) Let $p, \mathcal{C}_{p}$ and $\widehat{\mathcal{C}}_{p}$ be as specified.

First let $\widehat{p}=p$. Then $\mathcal{C}_{p}=\widehat{\mathcal{C}}_{p}$, because, for any profile $\left(A_{1}, \ldots, A_{n}\right) \in \mathbf{U}^{n}$, $p \in F\left(A_{1}, \ldots, A_{n}\right)$ is equivalent to $\widehat{p} \in \widehat{F}\left(A_{1}, \ldots, A_{n}\right)$ (using that $\widehat{\hat{p}}=p$ ), i.e., to $p \in \widehat{F}\left(A_{1}, \ldots, A_{n}\right)$.

Now let $\widehat{p}=\neg p$. To show that $\mathcal{C}_{p}=\left\{C \subseteq N: C \notin \widehat{\mathcal{C}_{p}}\right\}$, we consider any $C \subseteq N$, and prove that $C \in \mathcal{C}_{p}$ is equivalent to $C \notin \widehat{\mathcal{C}_{p}}$. By $\widehat{p}=\neg p, p$ is 
contingent, and so there exists a profile $\left(A_{1}, \ldots, A_{n}\right) \in \mathbf{U}^{n}$ such that $\{i: p \in$ $\left.A_{i}\right\}=C$. Now $C \in \mathcal{C}_{p}$ is equivalent to $p \in F\left(A_{1}, \ldots, A_{n}\right)$, which is equivalent to $\widehat{p} \in \widehat{F}\left(A_{1}, \ldots, A_{n}\right)$ (as in case 1 ), i.e., to $\neg p \in \widehat{F}\left(A_{1}, \ldots, A_{n}\right)$, hence to $p \notin$ $\widehat{F}\left(A_{1}, \ldots, A_{n}\right)$, and so to $C \notin \widehat{\mathcal{C}_{p}}$, as required.

To prove (the sufficiency part of) Theorem 3 , let $X$ be non-separable and minimally connected, and let $F: \mathbf{U}^{n} \rightarrow \mathbf{U}$ be unbiased. Let $\widehat{F}$ and $\widehat{p}$ (for any $p \in X$ ) be defined as in Lemma 7 . By part (c) of Lemma $7, \widehat{F}$ satisfies all conditions required in Theorem 1 . So $\widehat{F}$ is dictatorial by Theorem 1 , say with dictator $i$. Hence, by part (d) of Lemma 7, $i$ is under $F$ a dictator on $Z_{+}:=\{p \in X: \widehat{p}=p\}$ and an inverse dictator on $Z_{-}:=\{p \in X: \widehat{p}=\neg p\}$ $\left(=X \backslash Z_{+}\right)$. So $F$ is effectively dictatorial.

\section{An illustration}

To illustrate the generality of our result, we apply Theorem 1 to the aggregation of (strict) preferences, represented in the judgment aggregation model. We consider the agenda $X=\{x P y, \neg x P y \in \mathbf{L}: x, y \in K$ with $x \neq y\}$, where

- $\mathbf{L}$ is a predicate logic for representing preferences, with

- a two-place predicate $P$ (representing strict preference), and

- a set of constants $K=\{x, y, z, \ldots\}$ with $|K| \geq 3$ (representing alternatives).

- $A$ is consistent if and only if $A \cup Z$ is consistent in the standard sense of predicate logic, with $Z$ defined as the set of rationality conditions on strict preferences. ${ }^{9}$

For details of this construction, see Dietrich and List (2007) (also List and Pettit 2004). Each rational judgment set $A_{i} \subseteq X$ uniquely represents a strict (i.e., asymmetric, transitive and connected) preference relation $\succ_{i} \subseteq K \times K$, where, for any $x, y \in K, x P y \in A_{i}$ if and only if $x \succ_{i} y$. For example, if $K=\{x, y, z\}$, the preference relation $x \succ_{i} y \succ_{i} z$ is represented by the judgment set $A_{i}=\{x P y, y P z, x P z, \neg y P x, \neg z P y, \neg z P x\}$.

The agenda $X$ thus defined is non-separable. It is also minimally connected (take $Y=\{x P y, y P z, z P x\}$ in parts (i) and (ii) and $Z=\{x P y, y P z\}$, where $x, y, z \in K$ are distinct alternatives). So Theorems 1 and 3 apply (whereas Theorem 2 does not apply, as $X$ is not locally asymmetric, in fact not even asymmetric). Let us state Theorem 3 for this agenda:

Corollary $\mathbf{5}$ For the agenda $X=\{x P y, \neg x P y \in \mathbf{L}: x, y \in K$ with $x \neq y\}$, every unbiased aggregation function $F: \mathbf{U}^{n} \rightarrow \mathbf{U}$ is effectively dictatorial.

\footnotetext{
${ }^{9}$ Formally, $\quad Z$ contains $\left(\forall v_{1}\right)\left(\forall v_{2}\right)\left(v_{1} P v_{2} \quad \rightarrow \quad \neg v_{2} P v_{1}\right) \quad$ (asymmetry), $\left(\forall v_{1}\right)\left(\forall v_{2}\right)\left(\forall v_{3}\right)\left(\left(v_{1} P v_{2} \wedge v_{2} P v_{3}\right) \rightarrow v_{1} P v_{3}\right)$ (transitivity), $\left(\forall v_{1}\right)\left(\forall v_{2}\right)\left(\neg v_{1}=v_{2} \rightarrow\right.$ $\left.\left(v_{1} P v_{2} \vee v_{2} P v_{1}\right)\right)$ (connectedness) and, for any distinct constants $x, y \in K, \neg x=y$ (exclusiveness of alternatives).
} 
What does this mean in the language of preference aggregation? A judgment aggregation function $F: \mathbf{U}^{n} \rightarrow \mathbf{U}$ uniquely represents a social welfare function with universal domain (taking strict preferences as input and output). Unbiasedness, applied to such a social welfare function, becomes the condition that, for any pair of alternatives $x, y \in K$ and any two preference profiles $\left(\succ_{1}, \ldots, \succ_{n}\right),\left(\succ_{1}^{*}, \ldots, \succ_{n}^{*}\right)$ in the universal domain, if [for all individuals $i, x \succ_{i} y$ if and only if $\left.y \succ_{i}^{*} x\right]$ then $\left[x \succ y\right.$ if and only if $\left.y \succ^{*} x\right]$. Corollary 5 thus implies that every unbiased social welfare function with universal domain is effectively dictatorial. No unanimity (Pareto) condition is needed. Although this result could also be obtained in standard social choice theory (for example, via Wilson's (1972) result on social choice without the Pareto principle), the observation that it is a corollary of our new result on judgment aggregation should illustrate the result's generality. Interestingly, unlike Wilson's and Arrow's theorems, our result continues to hold even if the rationality conditions on preference relations are relaxed to acyclicity alone (giving up full transitivity and connectedness). The reason is that the agenda $X$, as specified above, remains non-separable and minimally connected in a modified predicate logic obtained by weakening the conditions in the set $Z$ above so as to capture acyclicity alone. ${ }^{10}$

\section{Concluding remarks}

In judgment aggregation, we face not only a trade-off between different conditions on an aggregation function for any given agenda (as in preference aggregation), but also a trade-off between these conditions and the generality of the agendas for which they can be met by an aggregation function.

We have proved three impossibility results. Two of them (Theorems 1 and 3) apply to all standard example agendas in the literature, including the agendas representing preference agggregation problems. This generality is surprising, as we do not impose systematicity, a condition often criticized as being too strong. We impose the weaker condition of unbiasedness: a May-type neutrality condition applied to each proposition-negation pair, without requiring neutrality across pairs.

Our results show that, for many agendas, unbiasedness leads to dictatorship, or at least to effective dictatorship. Our agenda conditions (non-separability, minimal connectedness, and in Theorem 2 also local asymmetry) are tight: agendas that violate any of them avoid the impossibility. Theorems 2 and 3 require no unanimity, monotonicity or other responsiveness condition, unlike many related impossibility results of social choice. But we retain full rationality of individuals and the group.

\footnotetext{
${ }^{10}$ Formally, $Z$ then contains, for any sequence of distinct constants $x_{1}, x_{2}, \ldots, x_{k} \in K$, the proposition $\left(x_{1} P x_{2} \wedge \ldots \wedge x_{k-1} P x_{k}\right) \rightarrow \neg x_{k} P x_{1}$ (no cycle over $x_{1}, x_{2}, \ldots, x_{k}$ ) and, for any distinct constants $x, y \in K, \neg x=y$ (exclusiveness of alternatives).
} 
Our results appear significant, as they imply that, in virtually all realistic judgment aggregation problems, any aggregation function with commonly accepted properties must favour some propositions over their negations.

\section{References}

Dietrich, F. (2006) Judgment Aggregation: (Im)Possibility Theorems. Journal of Economic Theory 126(1): 286-298

Dietrich, F. (2007) A generalised model of judgment aggregation. Social Choice and Welfare 28(4): 529-565

Dietrich, F. (forthcoming) The possibility of judgment aggregation on agendas with subjunctive implications. Journal of Economic Theory

Dietrich, F., List, C. (2007) Arrow's theorem in judgment aggregation. Social Choice and Welfare 29(1): 19-33

Dokow, E., Holzman, R. (2005) Aggregation of binary evaluations, Working paper, Technion Israel Institute of Technology

Gärdenfors, P. (2006) An Arrow-like theorem for voting with logical consequences. Economics and Philosophy 22(2): 181-190

Konieczny, S., Pino-Perez, R. (2002) Merging information under constraints: a logical framework, Journal of Logic and Computation 12(5): 773-808

List, C., Pettit, P. (2002) Aggregating Sets of Judgments: An Impossibility Result. Economics and Philosophy 18: 89-110

List, C., Pettit, P. (2004) Aggregating Sets of Judgments: Two Impossibility Results Compared. Synthese 140(1-2): 207-235

May, K. O. (1952) A set of independent, necessary and sufficient conditions for simple majority decision. Econometrica 20: 680-684

Mongin, P. (2005) Factoring out the impossibility of logical aggregation. Working paper, CNRS, Paris

Nehring, K., Puppe, C. (2002) Strategy-Proof Social Choice on SinglePeaked Domains: Possibility, Impossibility and the Space Between. Working paper, University of California at Davies

Nehring, K., Puppe, C. (2005) The Sructure of Strategy-Proof Social Choice. Part II: Non-dictatorship, Anonymity and Neutrality. Working paper, University of Karlsruhe

Nehring, K., Puppe, C. (2006) Consistent Judgement Aggregation: The Truth-Functional Case. Working paper, University of Karlsruhe

Pauly, M., van Hees, M. (2006) Logical Constraints on Judgment Aggregation. Journal of Philosophical Logic 35: 569-585.

Pettit, P. (2001) Deliberative Democracy and the Discursive Dilemma. Philosophical Issues 11: 268-299

van Hees, M. (2007) The limits of epistemic democracy. Social Choice and Welfare 28(4): 649-666

Wilson, R. (1972) Social Choice Theory without the Pareto Principle. Journal 
of Economic Theory 5: 478-486

Wilson, R. (1975) On the Theory of Aggregation. Journal of Economic Theory 10: 89-99 